\title{
Evaluación de los patrones de movimiento fundamentales en niños: comparación de género en escolares deEducación Primaria \\ Assessment of fundamental movement patterns in children: a gender comparison on Primary School students
}

\author{
Miguel García-Jaén, Sergio Sellés-Pérez, Juan Manuel Cortell-Tormo, Alberto Férriz-Valero, Roberto Cejuela \\ Universidad de Alicante (España)
}

\begin{abstract}
Resumen. El propósito del estudio fue valorar los patrones de movimiento fundamentales en niños y niñas de ocho y nueve años mediante el test Functional Movement Screen ${ }^{T M}$ (FMS), y establecer una comparación de género entre escolares de Educación Primaria. Para ello, 40 estudiantes de primaria (20 niñas y 20 niños) participaron en el estudio. La ejecución de cada participante se grabó digitalmente durante las clases de Educación Física en el colegio, y posteriormente fue analizada conjuntamente por dos evaluadores expertos en el uso del FMS. Finalmente, se procedió al análisis estadístico de los datos. Los resultados obtenidos mostraron que las niñas obtuvieron puntuaciones más altas en la suma de las siete pruebas, en comparación con los niños (15.3 \pm 1.999 vs. $13.75 \pm 1.970 ; p=.017)$. Particularmente, las niñas puntuaron significativamente mejor que los niños en el test de sentadilla profunda $(2.3 \pm .657$ vs. $2.75 \pm .444 ; p=.015)$ y en el test de estocada en línea $(1.8 \pm .834$ vs. $2.75 \pm .444 ; p=.000)$. En conclusión, las niñas obtuvieron una puntuación total en el test FMS superior a los niños, puntuando significativamente mejor que los niños en el test de sentadilla profunda y en el test de estocada en línea. Además, la mayoría de las niñas puntuaron por encima de 14 puntos, mientras que la mayoría de los niños puntuaron igual o por debajo de 14 puntos, lo cual podría indicar una menor competencia motriz y un riesgo incrementado de lesión en los niños.
\end{abstract}

Palabras clave. Competencia motriz, Movimiento Funcional, Calidad de Movimiento, FMS, Diferencias de Género, Niños, Educación Física, Educación Primaria.

\begin{abstract}
The aim of this study was to evaluate the fundamental movement patterns in eight and nine-year-old children, using the Functional Movement Screen ${ }^{T M}$ test (FMS) and analyzing gender differences in FMS scores among Primary Education students. 40 children, 20 girls and 20 boys, participated in this study. The performance of each participant on the FMS was digitally recorded during the Physical Education classes at school. After that, collected data were analyzed by two expert evaluators in FMS. Finally, a statistical data analysis was developed. The obtained results showed that females had higher total composite scores in the seven FMS test compared with males (15.3 \pm 1.999 vs. $13.75 \pm 1.970 ; p=.017$ ). Particularly, females scored significantly better than males in the deep squat test ( $2.3 \pm .657$ vs. $2.75 \pm .444 ; p=.015)$ and in the in-line lunge test (1.8 \pm .834 vs. $2.75 \pm .444$; $p=.000$ ). In conclusion, females obtained higher total composite score than the males in the FMS test, scoring significantly better than males in deep squat test and in the in-line lunge test. In addition, the majority of the females scored above 14 points, while most of the males scored 14 or less points on the FMS total composite score, which could be an indicative of less motor skills and also an increased risk of injury in males.

Keywords . Motor Skills, Functional Movement, Quality of Movement, FMS, Sex differences, children, Physical Education, Primary Education.
\end{abstract}

\section{Introducción}

En el ámbito escolar, la realización de cualquier actividad físicodeportiva dentro del Área de Educación Física exige al alumno unos mínimos de coordinación motriz, de condición física y de habilidades y destrezas básicas. Cuando se alcanza este nivel mínimo, el éxito en la tarea está prácticamente garantizado (García-Cantó, 2010). Sin embargo, si el niño no llega a alcanzar estas condiciones básicas de competencia motriz, no podrá disfrutar con los retos motores impuestos por esas tareas, provocando en el alumno la sensación de incompetencia motriz, y con ello, la posible desmotivación hacia la práctica e incluso el abandono de la misma (Ruiz-Pérez, 2000; Sánchez-Bañuelos, 1996). Estas experiencias motrices negativas del niño en el ámbito educativo pueden incidir en su propio autoconcepto, y podrían llegar a suponer un riesgo de alejamiento de la práctica de actividades físico-deportivas en el futuro adolescente y adulto (Kerr, Cotè̀, Hay \& Coteì-Laurence, 1998; Telford, 1998; White, 1995). Por ello, resulta fundamental durante las clases de Educación Física fortalecer la motivación de los alumnos hacia la práctica físicay, de esta manera, influir de forma indirecta en la adquisición de hábitos futuros de práctica deportiva de los alumnos durante su tiempo de ocio (Gutiérrez, 2000; Ruiz-Pérez, 1994a; Ruiz-Pérez, 1994b). Por esta razón, es esencial que el maestro de Educación Física, a la hora de plantear cualquier tarea motriz o reto motor en el centro escolar, analice el entorno y el contexto en el que se va a implementar, evaluando para ello los niveles de condición física, competencia motriz y calidad del movimiento de sus alumnos (Ruiz-Pérez, 2000).

Tradicionalmente, se han aplicado diferentes baterías de test con objeto de predecir y evaluar la condición física, la aptitud física motora

Fecha recepción: 05-02-18. Fecha de aceptación: 13-05-18 Miguel García-Jaén

m.garciajaen@ua.es y el nivel de competencia y destreza motriz del alumno en Educación Primaria, a partir de las capacidades físicas básicas (Gómez, Berral, Viana \& Berral, 2002; Martínez-Castañeda, 2011; Ruiz-Pérez, Navia, Ruiz, Ramón \& Palomo, 2016). Estas pruebas, que pretenden evaluar cuantitativamente factores que inciden en la ejecución del movimiento (la resistencia cardiovascular, la flexibilidad y la fuerza), no consideran sin embargo los factores cualitativos del movimiento, que son los determinantes del componente motor (equilibrio, coordinación, agilidad y velocidad), (Puerto, de la Rosa, Montaner, \& de la Rosa, 2002). Se ha visto, no obstante, que tanto el equilibrio como las capacidades coordinativas (coordinación dinámica general y coordinación visomotriz) están estrechamente relacionados con el esquema corporal y el control postural, capacidades perceptivo-motrices fundamentales que junto con las capacidades físicas básicas constituyen los factores de la condición física (Martínez-Castañeda, 2011; Puerto, et al., 2002). Por tanto, resulta necesario complementar las evaluaciones cuantitativas tradicionales con otros test cualitativos que resulten válidos y fiables para medir la calidad de movimiento global y valoren los patrones de movimientos fundamentales en los niños.

En este sentido, el test Functional Movement Screen ${ }^{\mathrm{TM}}$ (FMS) es un sistema de cribado válido, comúnmente usado para valorar la calidad del movimiento global, a partir de la evaluación de los patrones de movimiento fundamentales de un individuo (Cook, Burton \& Hogenboom, 2006a; 2006b). Estos autores sostienen además que la prueba es útil para describir la competencia motriz de base del individuo, así como para identificar sus limitaciones y asimetrías en los movimientos funcionales básicos en cualquier edad (Cook, Burton, Hoogenboom \& Voight, 2014a, 2014b). El FMS está compuesto de siete tareas que retan el control motor del sujeto, al desafiar la movilidad, la estabilidad y el equilibrio mediante la ejecución de patrones específicos y fundamentales de movimiento. Así pues, a través de estos siete test se pretende evidenciar tanto los desequilibrios bilaterales como la 
movilidad-estabilidad de cada segmento corporal involucrado. El uso del test FMS ha ido ganando además popularidad en los últimos años como herramienta predictiva válida del riesgo de lesiones, estableciendo que calificaciones de $14 \mathrm{o}$ menos puntos indican el umbral a partir del cual el riesgo de lesión en la práctica físico-deportiva se incrementa significativamente (Bonazza, Smuin, Onks, Silvis \& Dhawan, 2017). No obstante, otros estudios han cuestionado el valor predictivo de este test (Moran, Schneiders, Mason, \& Sullivan, 2017; Newton, et al., 2017). Con relación a la fiabilidad del test, un reciente metaanálisis ha demostrado que el test FMS tiene una fiabilidad intra-evaluador e interevaluadores considerada excelente (Bonazza, et al., 2017).

Hasta el momento, la mayoría de las investigaciones ha utilizado el test FMS en poblaciones adultas, activas, masculinas y/o femeninas. Sin embargo, su uso en niños y/o adolescentes, dentro del ámbito escolar, no está muy extendido y, además, apenas existe comparación de puntuaciones entre géneros. Dada la necesidad, por una parte, de medir la calidad de movimiento global y los patrones de movimientos fundamentales en los niños para así conocer su competencia motriz de base, y por otra, dada las diferencias de género establecidas en relación con la activación muscular y la fuerza, el control motor, la estabilidad del core, y las ratios de lesiones entre hombres y mujeres, resulta necesario plantear investigaciones que evalúen los patrones de movimiento fundamentales en niños en edad escolar, y que determinen además posibles diferencias de género en sus resultados. Por tanto, el propósito de este estudio fue valorar la competencia motriz básica en niños y niñas de ocho y nueve años, a través de una evaluación de los patrones de movimiento fundamentales mediante el uso del test FMS, y establecer una comparación de género entre escolares de Educación Primaria.

\section{Metodología}

\section{Participantes}

Una muestra seleccionada por conveniencia de 40 estudiantes (20 alumnas y 20 alumnos) pertenecientes a los cursos $3^{\circ}$ y $4^{\circ}$ de la etapa educativa de Educación Primaria realizaron los diferentes test del estudio (edad: $8.5 \pm$ .5 años; peso: $35.72 \pm 12.46 \mathrm{~kg}$; altura: $1.4 \pm$ .09 metros; IMC: $17.82 \pm 4.39$ ). Para ser incluidos en el estudio, los participantes no habían de haber sufrido lesiones musculoesqueléticas, de cabeza o espalda en los últimos seis meses y no debían presentar problemas vestibulares, visuales o de equilibrio, ni reportar cualquier tipo de discapacidad física o psíquica que condicionase la ejecución de las pruebas. Antes del comienzo de la toma de datos, se procedió a obtener la autorización del centro educativo para la realización del estudio. Asimismo, los tutores legales de los participantes firmaron previamente un consentimiento informado donde se exponían los fines de esta investigación, así como los métodos y procedimientos empleados en la misma. En la tabla 1 se muestran las características de los participantes sesgados por género.

\section{Instrumentos}

Los datos antropométricos de peso y altura se obtuvieron mediante el uso de una báscula (Avery Ltd. Model 3306 ABV) y de un estadiómetro portátil (Holtain Ltd.). Se utilizaron los siguientes materiales para poder llevar a cabo los diferentes test que componen la batería del FMS: una pica de 1.22 metros, dos picas

Tabla 1.
\begin{tabular}{ccc} 
Datos de los participantes por género & \\
\hline & Niños & Niñas \\
\hline$n$ & 20 & 20 \\
\hline Edad (años) & $8.5 \pm .5$ & $8.5 \pm .5$ \\
Altura (m) & $1.40 \pm .10$ & $1.41 \pm .09$ \\
Peso $(\mathrm{Kg})$ & $35.91 \pm 14.31$ & $35.52 \pm 10.66$ \\
IMC & $17.94 \pm 5.14$ & $17.69 \pm 3.61$ \\
\end{tabular}

de .61 metros, un tablón de madera de 2x6 cm, una cinta adhesiva, una cinta métrica y unos conos. Además de ello, se emplearon dos cámaras de alta definición con tecnología de grabación4K paragrabar la ejecución de los ejercicios desde los planos frontal y sagital.

\section{Procedimiento}

Tras la selección de los participantes usando un informe de antecedentes médicos que habían cumplimentado previamente los tutores legales, se procedió a grabar digitalmente la ejecución de los ejercicios de cada participante durante las sesiones de Educación Física. Las evaluaciones se llevaron a cabo de manera individual, realizándose una sola vez el test por alumno. El FMS consiste en siete movimientos y tres pruebas aclaratorias de dolor. En el test se incluyen las siguientes tareas: sentadilla profunda, paso de valla, estocada en línea, movilidad de hombro, elevación activa de la pierna recta, estabilidad del tronco en flexiones y estabilidad rotatoria. En cinco de las siete pruebas (todas a excepción de la sentadilla profunda y la estabilidad del tronco en flexiones) se debe evaluar el rendimiento tanto del lado corporal izquierdo como del lado
Tabla 2.

\begin{tabular}{|c|c|}
\hline Ejercicio & Instrucción \\
\hline \multirow{6}{*}{ Sentadilla profunda } & $\begin{array}{l}\text { Ponte de pie con los pies separados aproximadamente a la altura de los hombros y los dedos de los pies } \\
\text { apuntando hacia delante. }\end{array}$ \\
\hline & $\begin{array}{l}\text { Sujeta la pica con ambas manos y colócala en la parte superior de tu cabeza para que tus hombros y tus } \\
\text { codos estén flexionados. }\end{array}$ \\
\hline & Presiona la pica para que esté directamente sobre tu cabeza \\
\hline & Mientras mantienes el cuerpo recto y los talones y la pica en posición. \\
\hline & Desciende hacia abajo tanto como te sea posible. \\
\hline & Aguanta abajo durante un segundo antes de volver a la posición inicial. \\
\hline \multirow{4}{*}{ Paso de valla } & Ponte de pie con los pies juntos y los dedos de los pies tocando el tablón de 2x6. \\
\hline & Sujeta la pica con ambas manos y colócala detrás de tu cuello y espalda. \\
\hline & $\begin{array}{l}\text { Mientras mantienes una postura recta, levanta la pierna derecha y pisa el obstáculo, haciendo seguro } \\
\text { levantar el pie hacia la espinilla y mantén el pie en línea con el tobillo, rodilla y cadera. }\end{array}$ \\
\hline & $\begin{array}{l}\text { Toca el suelo con el talón y vuelve a la posición inicial mientras mantienes el pie en línea con el } \\
\text { tobillo, la rodilla y la cadera. }\end{array}$ \\
\hline \multirow{9}{*}{ Estocada en línea } & $\begin{array}{l}\text { Coloca la pica a lo largo de la espalda para que toque la parte posterior de la cabeza, la parte superior } \\
\text { de la espalda, }\end{array}$ \\
\hline & y tu sacro (parte baja de la espalda). \\
\hline & $\begin{array}{l}\text { Mientras sujetas la pica, tu mano derecha debe estar contra la parte posterior de su cuello, y la mano } \\
\text { izquierda debe estar contra la parte inferior de la espalda. }\end{array}$ \\
\hline & Ingresa al tablón de $2 \times 6$ con un pie plano y recto y tu dedo gordo en la marca cero. \\
\hline & El talón izquierdo debe colocarse en la marca de medición de la inserción del tibial. \\
\hline & Ambos dedos de los pies deben estar apuntando hacia adelante, con los pies plano. \\
\hline & $\begin{array}{l}\text { Mantén una postura recta para que la pica permanezca en contacto con la cabeza, la parte superior de la } \\
\text { espalda }\end{array}$ \\
\hline & $\begin{array}{l}\text { y la parte superior de las nalgas, desciende a una posición de zancada para que la rodilla derecha toque } \\
\text { el tablón de } 2 \times 6 \text { detrás del talón izquierdo/derecho. }\end{array}$ \\
\hline & Vuelve a la posición inicial \\
\hline
\end{tabular}

Tabla 3.

Instrucciones verbales durante la ejecución de cada ejercicio del test FMS. Parte 2. (adaptado de Cook, 2010) ***

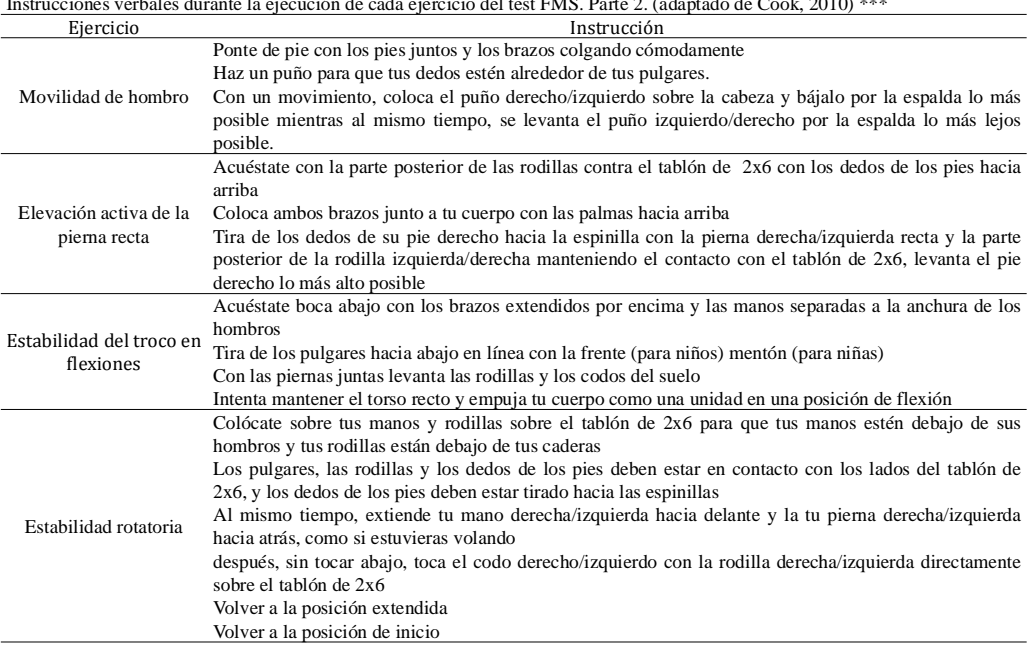


derecho. Los participantes completaron cinco minutos de calentamiento dirigido, compuesto por ejercicios básicos de movilidad dinámica, antes de la ejecución de los ejercicios. Se les facilitó a los participantes instrucciones verbales de cada ejercicio siguiendo las pautas de descripción propuestas por Cook (2010), (Tabla 1 y Tabla 2), permitiéndoles la realización de tres intentos de ejecución por ejercicio. La máxima puntuación por ejercicio es de tres puntos por ejercicio, siendo por tanto 21 puntos la máxima puntuación que una persona puede obtener en el test. Se consiguen tres puntos si la persona es capaz de realizar el movimiento, cumpliendo todos los criterios establecidos sin necesidad de aplicar una compensación. Dos puntos si consigue realizar el movimiento sin necesidad de aplicar una compensación, aunque sin cumplir todos los criterios establecidos. Se otorga un punto si el individuo es capaz de realizar el movimiento, pero sin cumplir todos los criterios establecidos y con la asistencia de una ayuda o compensación. Se obtiene un cero si la persona no puede completar el ejercicio y/o siente dolor al realizarlo (Cook, et al., 2006a). En los movimientos que implican ejercicios unilaterales se valora independientemente la extremidad izquierda y derecha, tomándose como referencia la puntuación más baja en el cálculo de la puntuación total del test. Las pruebas aclaratorias de dolor fueron puntuadas como positivo (presenta dolor) o negativo (no presenta dolor) en función de si presentaban dolor para el alumno. Si la persona presenta dolor durante el movimiento, automáticamente obtiene una puntuación de cero en dicha prueba, independientemente de la puntuación obtenida anteriormente (Cook, et al., 2006a).

La ejecución de cada participante se grabó digitalmente desde diferentes planos de movimiento y posteriormente fue analizada conjuntamente por dos evaluadores con al menos dos años de experiencia en el uso de FMS (Anderson, Neumann, \& Huxel Bliven, 2015).

\section{Análisis estadístico de los datos}

Los dados descriptivos del estudio (edad, talla, peso, e IMC) se muestran como la media \pm la desviación estándar. El análisis estadístico inferencial de los datos se realizó utilizando un modelo general linear para medidas repetidas mediante el análisis de la varianza (ANOVA), usando el software estadístico SPSS, versión 21.0 (Chicago, IL, USA). El nivel de significación estadística se estableció en $p<.005$.

\section{Resultados}

La figura 1 muestra de forma descriptiva el resultado obtenido, en valores porcentuales, por el total de la muestra en cada una de las siete pruebas. Las pruebas en las que mayormente se obtuvo la máxima puntuación (3 puntos) fueron la de estabilidad del tronco en flexiones (65\%), la de sentadilla profunda (57,5\%), y la prueba del paso de valla ( $50 \%$ izquierda; $55 \%$ derecha; $42,5 \%$ total). Las pruebas que mayormente puntuaron con 2 puntos fueron la de elevación activa de la pierna recta (60\% izquierda; $50 \%$ derecha; $65 \%$ total) y la de estocada en línea (55\% izquierda; $40 \%$ derecha; $45 \%$ total). Finalmente, las pruebas en las que se obtuvo solamente 1 punto fueron las dos pruebas restantes: estabilidad rotatoria (60\% izquierda; 70\% derecha; 70\% total) y movilidad del hombro (30\% izquierda; 55\% derecha; 55\% total). Las tres pruebas aclaratorias de dolor resultaron negativas en todos los participantes.

La figura 2 muestra la comparativa por género de las puntuaciones medias totales obtenidas por los participantes. Las niñas obtuvieron una puntuación total estadísticamente significativa superior en el test FMS que los niños (13.8 \pm 2.0 vs. $15.3 \pm 2.0 ; p=.017)$.

La figura 3 muestra el porcentaje de estudiantes por género que puntuaron por debajo o igual a 14 (riesgo incrementado de lesión) y por encima de 14 (riesgo disminuido de lesión) en el test FMS. Los resultados muestran que un $75 \%$ de las niñas $(n=15)$ y un $30 \%$ de los niños $(n=6)$ puntuaron por encima de 14 puntos, mientras que un $25 \%$ de las niñas $(n=5)$ y un $70 \%$ de los niños $(n=14)$ puntuaron igual o por debajo de 14 puntos.

En la tabla 4 se observan las diferencias por género en cuanto a las puntuaciones obtenidas en cada una de las siete pruebas que componen

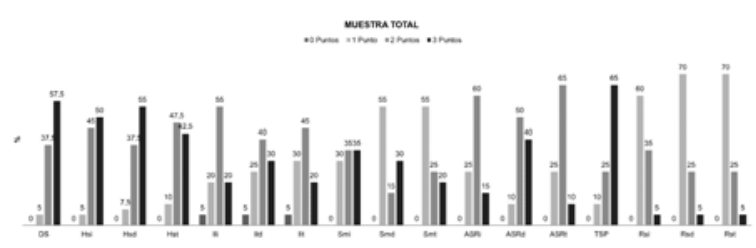

DP: Sentadilla Profunda; HSi: Paso de Valla iqquierda; HSd: Paso de Valla derecha; HSt: Paso de Valla

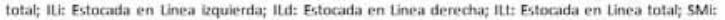
Movilidad del Hombro izquierda; SMd: Movildad del Hombro derecha; SMt: Movildad del Hombro total: ASRl: Elevación Activa de la Plerna izquierda: ASRd: Elevación Activa de la Plerna derecha: ASRt: Flevacion Activa de ta Plerna total; TSP: Estabilidad del Tronco en Flexianes; RS: Estabilidad Giratoria iq quierda: RSd: Estabilidad Giratoria derecha; RSt: Estabilidad Giratoria total.

Figura 1. Porcentaje de puntuación obtenida por el total de la muestra para cada una de las 7 pruebas del test FMS.

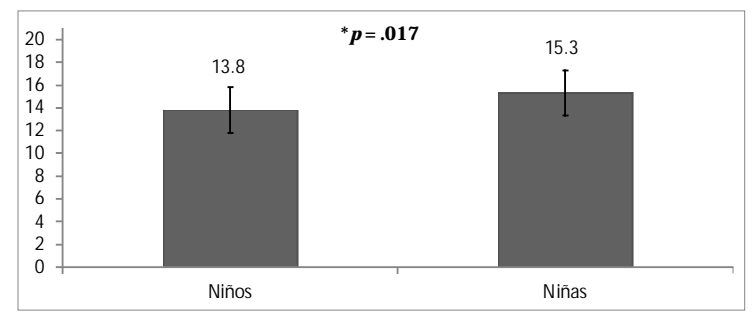

Figura 2. Comparativa de la puntuación total media obtenida en el test FMS en estudiantes de segundo ciclo de primaria por género.

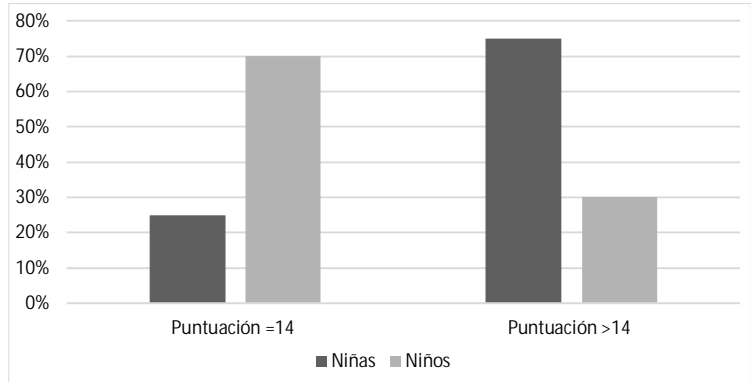

Figura 3. Porcentaje de estudiantes de segundo ciclo de primaria por género que puntuaron por debajo/igual a $14 \mathrm{y}$

el test FMS. Particularmente las niñas puntuaron de manera estadísticamente significativa más alto que los niños en el test de sentadilla profunda (2.3 \pm .8 vs. $2.8 \pm .4 ; p=.000$ ). Lo mismo sucede en el test de estocada en línea, puesto que en este ejercicio tanto con la pierna izquierda (1.9 \pm .8 vs. $2.8 \pm .4 ; p=.000)$, como con la pierna derecha (2.0 \pm .9 vs. $2.8 \pm .4 ; p=.000)$ y con el promedio total de las dos (1.8 \pm .8 vs. $2.8 \pm .4 ; p=.000$ ), las niñas obtuvieron una puntuación superior estadísticamente significativa en comparación los niños. En el resto de los ejercicios no se aprecian diferencias significativas en la puntuación entre niños y niñas.

En el género femenino los ejercicios en los que las participantes obtuvieron un mejor resultado fueron la estocada en línea (2.8 \pm .4) y la

Tabla 4

Comparativa de las puntuaciones en los diferentes ejercicios del test FMS en estudiantes de segundo ciclo de primaria por género.

\section{Sentadilla profunda}

Paso de valla (I)

Paso de valla (D)

Paso de valla (T)

Estocada en línea (I)

Estocada en línea (D)

Estocada en línea (T)

Movilidad de hombro (I)

Movilidad de hombro (D)

Movilidad de hombro (T)

Elevación activa de pierna recta (I)

Elevación activa de pierna recta (D)

Elevación activa de pierna recta (T)

Estabilidad del tronco en flexiones

Estabilidad rotatoria (I)

Estabilidad rotatoria (D)

Estabilidad rotatoria (T)

$\mathrm{I}=$ izquierdo/a; $\mathrm{D}=$ derecho/a; $\mathrm{T}=$ total

$\mathrm{I}=\mathrm{izquier}$
$* p<.005$

$* p<.005$
$* * *$
$*<.001$ 
sentadilla profunda (2.8 \pm .4$)$, mientras que en el género masculino la prueba en la que se obtuvo una mayor puntuación media fue la estabilidad del tronco en flexiones (2.6 \pm .7). En ambos géneros la peor puntuación media se observa en el ejercicio de estabilización rotatoria.

\section{Discusión}

El propósito de este estudio fue conocer la competencia motriz básica en escolares de ocho y nueve años de Educación Primaria, mediante la valoración de los patrones de movimiento fundamentales a través del uso del test FMS, e investigar posibles diferencias de género existentes entre niñas y niños. Los resultados del estudio mostraron que solamente en tres de las siete pruebas la mayoría de los participantes obtuvo la máxima puntuación (test de estabilidad de tronco en flexiones, de sentadilla profunda y de paso de valla). En las restantes pruebas los participantes puntuaron mayormente con dos (test de elevación activa de la pierna recta y estocada en línea) o un punto solamente (test de estabilidad rotatoria y movilidad del hombro). Estos resultados indican diferencias en la competencia motriz básica de los escolares en función del patrón de movimiento específico evaluado, y pueden revelar por tanto posibles limitaciones de movilidad, estabilidad, coordinación y equilibrio en función de la prueba valorada.

El test FMS se creó para valorar los patrones de movimiento fundamentales y puede medir de forma indirecta determinados factores intrínsecos tales como la activación y fuerza muscular, el control neuromotor, el equilibrio, la flexibilidad y la capacidad de estabilización del core (Cook, 2010). Tales factores intrínsecos, junto a otros extrínsecos como el género, la edad o el nivel madurativo del sujeto pueden determinar el nivel de competencia motriz básica del niño e influir por tanto en sus puntuaciones del test FMS, lo que nos permite llegar a identificar las posibles limitaciones y asimetrías que presenta ese sujeto en la ejecución de movimientos fundamentales que, en última instancia, se realizan tanto en la vida diaria en general como específicamente en las actividades físico-deportivas en el ámbito escolar (Anderson, et al., 2015; Cook, et al., 2006a, 2006b; Kiesel, Plisky, \& Butler, 2011).

El principal hallazgo de nuestra investigación surgió al realizar la comparación de género entre los escolares participantes en el estudio. Los resultados mostraron que, en la suma de las siete pruebas del test FMS, las niñas obtuvieron una puntuación total estadísticamente significativa superior a los niños (15.3 \pm 1.999 vs. $13.75 \pm 1.970 ; p=.017)$ Particularmente, las niñas puntuaron significativamente mejor que los niños en el test de sentadilla profunda $(p=.000)$ y en el test de estocada en línea, tanto con la pierna izquierda y derecha $(p=.000)$, como con el promedio total de las dos $(p=.000)$. En el resto de los ejercicios no se apreciaron diferencias significativas en la puntuación en función del género. Este hallazgo evidencia que las niñas tuvieron un rendimiento total en el test FMS superior que los niños, lo cual indica diferencias en la evaluación de los patrones de movimiento fundamentales y, por tanto, diferencias en el nivel de competencia motriz de este grupo de escolares. Estos hallazgos podrían revelar y evidenciar déficits en la capacidad de movimiento funcional en los niños y un incremento del riesgo de lesión en la práctica motriz de estos escolares. Los factores contribuyentes a ese mayor riesgo de lesión podrían estar relacionados con factores intrínsecos (déficits en la movilidad, en la estabilización del core y en la capacidad de coordinación y equilibrio), además de estar relacionados con factores extrínsecos como la edad, el género y el proceso de maduración biológica del niño.

Hasta donde alcanza nuestro conocimiento, este es el primer estudio en utilizar el test FMS para evaluar la competencia motriz de escolares de Educación Primaria de ocho y nueve años. Además, es el primer estudio en realizar una comparación de género con el test FMS en una población pediátrica, así como en identificar y evidenciar diferencias de rendimiento en el test entre niñas y niños, tanto en la puntuación total como en la puntuación de algunas pruebas en particular. En consecuencia, la comparación de nuestros resultados con otros hallazgos en la literatura relacionada es limitada. Otros estudios habían realizado ya comparaciones de género, pero en otras poblaciones, de diferente edad y niveles de actividad y condición física, y con resultados diferentes a los de nuestro estudio. Anderson et al. (2015) investigaron las diferencias de género en las puntuaciones del test FMS en 60 escolares adolescentes (31 chicos y 29 chicas) de Educación Secundaria (16 \pm 1.1 años), reportando que las chicas puntuaron en la suma total de las siete pruebas significativamente más bajo que los chicos $(p=.004)$, así como en los test específicos de estocada en línea $(p<.004)$ y de estabilidad del tronco en flexiones $(p=.001)$, y sugieren que por tanto podrían tener menor competencia motriz y un riesgo aumentado de lesión. Por su parte, Schneiders, Davidsson, Hörman, \& Sullivan, (2011) evaluaron a 209 jóvenes activos (108 chicas y 101 chicos de entre 18-40 años -21.9 \pm 3.7 años-) y no encontraron diferencias significativas entre sexos en las puntuaciones totales del FMS (chicas 15.6 puntos y chicos 15.8 puntos). En línea con ese estudio, Paszkewicz, McCarty, \& Van Lunen (2013) compararon diferentes test estáticos y funcionales entre atletas jóvenes (11.45 \pm 1.98 años) y reportaron que las puntuaciones totales del test FMS incrementaron significativamente entre aquellos participantes que eran prepúberes y los que eran postpúberes, sin encontrar no obstante diferencias significativas en la comparación entre chicas y chicos.

Nuestros resultados muestran, a diferencia de estas investigaciones, que fueron las niñas de ocho y nueve años quienes obtuvieron mayores puntuaciones totales en el test FMS que los niños. Esto podría atribuirse parcialmente a diferencias demográficas entre participantes, fundamentalmente en relación con la edad, los niveles de actividad y condición física, y a los procesos y niveles específicos de maduración biológica de cada muestra. Nosotros hemos evaluado a una muestra específica de escolares de Educación Primaria (8.5 \pm .5 años), mientras que los anteriores estudios evaluaron a participantes que eran mayores que los de nuestro estudio (adolescentes de secundaria ( $16 \pm 1.1$ años); jóvenes activos (21.9 \pm 3.7 años); o jóvenes atletas (11.45 \pm 1.98 años). Esto sugiere que tanto la competencia motriz de base como los patrones de movimiento fundamentales serían dinámicos y estarían influenciados por factores extrínsecos como la edad, el nivel de condición física y el proceso madurativo, además de los pertinentes factores intrínsecos del individuo. Por tanto, debido a los cambios producidos en la movilidad, el control motor, la coordinación, el equilibrio, la fuerza y la flexibilidad a lo largo de todo el período de crecimiento y maduración personal, no resultaría apropiado establecer comparaciones y extrapolar los resultados del test FMS a diferentes poblaciones de distinta edad y nivel de actividad y condición física. Futuras investigaciones deberían tener en cuenta esta consideración a la hora de establecer valores y datos normativos sobre FMS en diferentes rangos de edad.

Otro de los hallazgos principales de nuestro estudio fue que la mayoría de las niñas de ocho y nueve años ( $75 \%$; $n=15)$, y tan sólo un número reducido de los niños de esa edad $(30 \% ; n=6)$ puntuaron por encima de 14 puntos, mientras que un $25 \%$ de las niñas $(n=5)$ y un $70 \%$ de los niños $(n=14)$ puntuaron igual o por debajo de 14 puntos. La literatura científica ha sugerido que una puntuación total de 14 puntos o menos es indicativo de un riesgo incrementado de lesión (Bonazza, et al., 2017; Chorba, Chorba, Bouillon, Overmyer, \& Landis, 2010). Si aplicamos este criterio a los resultados de nuestro estudio, podemos determinar que la mayoría de los niños evaluados podrían tener un riesgo incrementado de lesión durante la práctica motriz, tanto de las actividades físico-deportivas desarrolladas dentro como fuera del ámbito escolar, mientras que el riesgo de lesión en la mayoría de las niñas evaluadas se presentaría como riesgo disminuido. Estos hallazgos, de confirmarse en futuros estudios que evalúen la competencia motriz básica de escolares a lo largo de todo el ciclo educativo de Educación Primaria, deberían ser considerados por el maestro de Educación Física a la hora de analizar el entorno y el contexto donde plantear e implementar cualquier tarea motriz, siguiendo el criterio de Ruiz-Pérez (2000) y ser por tanto capaz de detectar deficiencias en la capacidad motriz del alumno, así como de plantear retos motores que ayuden a mejorar los patrones fundamentales de movimiento y restaurar una óptima capacidad de movimiento funcional, mejorando en última instancia su competencia motriz (Anderson, et al., 2015). No obstante, hay que señalar que 
estudios recientes han cuestionado el valor predictivo de este test para determinar el riesgo de lesión (Moran, etal., 2017; Newton, et al., 2017), y que, en todo caso, ese umbral de 14 puntos no ha sido establecido como válido para una población de escolares de ocho y nueve años, por lo que el riesgo real de lesión es desconocido y puede tan solo inferirse a partir de investigaciones previas que han examinado esta cuestión en otros grupos de población (Bonazza, et al., 2017), por lo que nuestras conclusiones en este sentido son limitadas.

En relación a otras limitaciones del estudio, es de destacar que esta investigación no examinó directamente los factores intrínsecos que pueden influir en la competencia motriz básica y en la ejecución de los patrones de movimiento fundamentales (actividad y fuerza muscular, control motor, coordinación, equilibrio y estabilidad del core), por lo que solamente podemos especular e inferir las razones por las que los niños puntuaron más bajo que las niñas, y relacionarlo de forma limitada con factores extrínsecos como la edad, el nivel de actividad y condición física, el género y el proceso madurativo del niño. De todas formas, a pesar de que estos factores intrínsecos no son medidos directamente por el test FMS, está generalmente aceptado que se requiere de la integración de todos esos factores intrínsecos para completar con éxito las diferentes pruebas del test FMS (Cook, et al., 2014a; 2014b). Resultaría interesante que futuras investigaciones explorasen las posibles relaciones entre las puntuaciones del test FMS y los niveles de activación muscular, control motor, coordinación, equilibrio y capacidad estabilizadora del core. Asimismo, se podrían plantear más estudios que indaguen las posibles relaciones entre los niveles de competencia motriz, medidos con el test FMS, con los niveles autopercibidos de condición física, actividad física y calidad de vida relacionada con la salud, y establecer comparaciones de género en escolares deEducación Primaria.

\section{Conclusiones}

Los resultados mostraron que, en la suma de las siete pruebas del test FMS, las niñas obtuvieron una puntuación total estadísticamente significativa superior a los niños, puntuando significativamente mejor que los niños en el test de sentadilla profunda y en el test de estocada en línea. En el resto de los ejercicios no se apreciaron diferencias significativas en la puntuación en función del género. Además, la mayoría de las niñas de ocho y nueve años, y tan sólo un número reducido de los niños de ocho y nueve años puntuaron por encima de 14 puntos, mientras que la mayoría de los niños puntuaron igual o por debajo de 14 puntos, lo cual podría indicar una menor competencia motriz y un riesgo incrementado de lesión en los niños. Los resultados de la muestra total indican diferencias en la competencia motriz básica de los escolares en función del patrón de movimiento específico evaluado, y pueden revelar por tanto posibles limitaciones de movilidad, estabilidad, coordinación y equilibrio en función de la prueba valorada.

\section{Agradecimientos}

Miguel García-Jaén participó en este estudio subvencionado por un contrato predoctoral de la Generalitat Valenciana (ACIF/2016/048). Los autores desean agradecer al centro educativo y a los estudiantes su participación voluntaria en este estudio. Finalmente, los autores desean mostrar su agradecimiento al grupo DOGESPORT por la posibilidad de presentar los resultados preliminares de este estudio en el IV Congreso Internacional en Optimización del Entrenamiento y Readaptación Físico-Deportiva.

\section{Referencias}

Anderson, B. E., Neumann, M. L., \& Huxel Bliven, K. C. (2015). Functional movementscreen differences betweenmaleand female secondary school athletes. Journal of Strength and Conditioning Research, 29(4), 1098-1106.

Bonazza, N. A., Smuin, D., Onks, C. A., Silvis, M. L., \& Dhawan, A. (2017). Reliability, validity, and injury predictive value of the functional movement screen: a systematic review and meta-analysis. The American Journal of Sports Medicine, 45(3), 725-732.

Chorba, R. S., Chorba, D. J., Bouillon, L. E., Overmyer, C. A., \& Landis, J. A. (2010). Use of a functional movement screening tool to determine injury risk in female collegiate athletes. North American journal of sports physical therapy: NAJSPT, 5(2), 47.

Cook, G. (2010). Movement: functional movement systems; screening, assessment and corrective strategies. On Target Publications.

Cook, G., Burton, L., \& Hoogenboom, B. (2006a). Pre-participation screening: the use of fundamental movements as an assessment of function - part 1. North American Journal of Sports Physical Therapy: NAJSPT, 1(2), 62.

Cook, G., Burton, L., \& Hoogenboom, B. (2006b). Pre-participation screening: the use of fundamental movements as an assessment of function - part 2. North American Journal of Sports Physical Therapy: NAJSPT, 1(3), 132-9.

Cook, G., Burton, L., Hoogenboom, B. J., \& Voight, M. (2014a). Functional movement screening: the use of fundamental movements as an assessment of function part 1. International journal of sports physical therapy, 9(3), 396.

Cook, G., Burton, L., Hoogenboom, B. J., \& Voight, M. (2014b). Functional movement screening: the use of fundamental movements as an assessment of function part 2. International journal of sports physical therapy, 9(4), 549.

García Cantó, E. (2010). Niveles de actividad física habitual en escolares de 10 a 12 años de la Región de Murcia. Tesis Doctoral. Universidad de Murcia.

Gómez, J., Berral, C., Viana, B., \& Berral, F (2002). Valoración de la aptitud física en escolares. Archivos de medicina del deporte, XIX, 273-282.

Gutieìrrez, M.(2000). Actividad fiìsica, estilos de vida y calidad de vida. Revista de Educacioin Fìisica, 77, 5-14.

Kerr, R., Coteì, J., Hay, J. \& Coteì-Laurence, P. (1998). Changing attitudes and expectation in a university setting: a case study. Physical educator, 55(3), 160168.

Kiesel, K., Plisky, P. \& Butler R. (2011). Functional movement test scores improve following a standardized off-season intervention program in professional football players. Scandinavian Journal of Medicine and Science in Sports. 21:287-292.

Martínez Castañeda, R. (2011). Valoración de la condición física en relación con la salud en escolares preadolescentes de la provincia de León: influencia de la actividad física en el sobrepeso, obesidad y riesgo de síndrome metabólico. Tesis Doctoral. Universidad de León.

Moran, R. W., Schneiders, A. G., Mason, J., \& Sullivan, S. J. (2017). Do Functional Movement Screen (FMS) composite scores predict subsequent injury? A systematic review with meta-analysis. British Journal of Sports Medicine, 51(23), 1661-1669.

Newton, F., McCall, A., Ryan, D., Blackburne, C., aus der Fünten, K., Meyer, T., ... \& McCunn, R. (2017). Functional Movement Screen(FMS ${ }^{\mathrm{TM}}$ ) score does not predict injury in English Premier League youth academy football players. Science and Medicine in Football, 1(2), 102-106.

Paszkewicz, J. R., McCarty, C. W., \& Van Lunen, B. L. (2013). Comparison of functional and static evaluation tools among adolescent athletes. The Journal of Strength \& Conditioning Research, 27(10), 2842-2850.

Puerto, J. R. G., de la Rosa, C. J. B., Montaner, B. H. V., \& de la Rosa, F. J. B. (2002). Valoración de la aptitud física en escolares. Archivos de medicina del deporte: revista de la Federación Española de Medicina del Deporte y de la Confederación Iberoamericana de Medicina del Deporte,(90), 273-282.

Ruiz Pérez, L. M. (1994a). Desarrollo Motor y Actividades Fiìsicas (3a ed.). Madrid: Gymnos.

Ruiz Pérez, L. M. (1994b). Deporte y Aprendizaje: Procesos de adquisicioin y desarrollo de habilidades. Madrid: Visor.

Ruiz Pérez, L. M. (2000). Aprender a ser incompetente en educacioìn fiìsica: un enfoque psicosocial. Apunts. Educacioìn fiìsica y Deportiva, 60, 20- 25.

Ruiz-Pérez, L. M., Navia Manzano, J.A., Ruiz Amengual, A., Ramón Otero, I., \& Palomo Nieto, M. (2016). Coordinación motriz y rendimiento académico en adolescentes. RETOS. Nuevas Tendencias en Educación Física, Deporte y Recreación,(29).

Saìnchez Banpuelos, F. (1996). La actividad fì̀sica orientada hacia la salud. Madrid: Biblioteca Nueva.

Schneiders, A. G., Davidsson, Å., Hörman, E., \& Sullivan, S. J. (2011). Functional movement screen ${ }^{\mathrm{TM}}$ normative values in a young, activepopulation. International journal of sports physical therapy, 6(2), 75.

Telford, A. (1998). Physical education teacher is ayadic interaction with high and low motor skilled student in secondary school. Microform Publication. Oregon: Universidad de Oregon.

White, S. A. (1995). The perceived purposes of sport among male and female intercollegiate and recreational sport participants. International Journal of Sport Psychology, 26, 490-502. 